\title{
GAs Based Steady State Analysis of Parallel-Operated Three Phase Self-Excited Induction Generators
}

\author{
A.I. Alolah M.A. Alkanhal \\ EE Dept.- College of Eng.- King Saud University, P.O.Box 800, Riyadh 11421, Saudi Arabia \\ Phone no.: +96614676798, Fax: +96614676757, E-mail: alolah@ksu.edu.sa
}

\begin{abstract}
This paper proposes a scheme for a steady state analysis for a group of three phase self-excited induction generators operating in parallel. The proposed scheme to solve for the unknowns is a sequential numerical optimizer. This optimizer consists of two parts based on genetic algorithm (GA) is used to bring the values of the unknowns close to the region of the (optimality) solution Unlike the other reported methods of analysis, the same equation is to be solved irrespective of the unknown parameters. The analysis presented is validated by experimental results of two generators connected in parallel.
\end{abstract}

Key words: induction generator, parallel operation, steady state, self excitation, genetic algorithms.

\section{List of Symbols \\ $F, u \quad$ p.u frequency and speed, respectively \\ $C, X_{c} \quad$ per phase value of excitation capacitance $(\mu F)$ and \\ its p.u reactance (at base frequency), respectively $X_{s}, X_{r}, X_{L} \quad \begin{aligned} & \text { p.u stator, and rotor leakage and load reactances at } \\ & \text { base frequency, respectively }\end{aligned}$ \\ $X_{m}, X_{o} \quad$ p.u saturated and unsaturated magnetizing \\ reactances at base frequency, respectively \\ $V_{g}, V_{o} \quad$ p.u. air gap and terminal voltages, respectively \\ $I_{c}, I_{l}, I_{s} \quad$ p.u. per phase excitation capacitance, load and \\ G, // Generator and parallel, respectively}

\section{Subscript}

$i \quad \mathrm{i}^{\text {th }}$ generator

\section{Introduction}

It is well known that an externally driven induction machine can sustain self-excitation when an appropriate value of a capacitor bank is properly connected across its stator terminals [1]. Brushless construction with squirrelcage rotor, reduced size, absence of DC supply for excitation, reduced maintenance cost, and better transient performances are the main advantages of induction generators over the synchronous generators. In recent years, self-excited induction generators have been employed as suitable isolated power sources in small hydroelectric and wind energy applications [1-2]. Steady state analyses of stand alone self-excited induction generator (SEIG) are well documented in the literature [1-7].

In wind power generating systems, physical size of the individual machines, operating at maximum efficiency, normal routine maintenance, future growth and reliability are the reasons to be operated in parallel. The model used to steady state analysis self-excited induction generators is the per-phase equivalent circuit approach that includes the loop-impedance method and the nodal admittance method [2,8]. Analyses of SEIG under such a mode of operation have been dealt with recently [8-10]. In the analyses reported, Newton-Raphson method has been utilized in to solve $(n+1)$ nonlinear equations. This method needs very near initial values to start the solution.

Genetic algorithms (GAs) are stochastic optimization techniques. They are simple, powerful, general purpose, derivative-free stochastic global optimization techniques (search algorithms) inspired by the laws of natural selection and genetics [7]. They follow Darwin's theory of evolution, where fitter individuals are likely to survive in a competing environment. These algorithms are derivative-free in the sense that they do not need functional derivative information to search for a set solution that minimizes (or maximizes) a given objective function. This property of GAs reduces the computational burden and search time, and also enables them to solve complex objective functions.

This paper deals with a new approach for the analysis of a three phase self-excited induction generators under parallel operation using GAs. This problem is formulated as a numerical optimization problem where no detailed derivation of analytical equations is needed. A GA initialized-Gradient optimizer is utilized to minimize a cost function of the total impedance or admittance equations of the circuit of the generator to obtain the frequency and other performance of the machine. Unlike the other reported methods of analysis, the same equations are to be solved irrespective of the unknown parameters. The equations can be solved for voltage and frequency, frequency and minimum value of excitation capacitors or frequency and speed of the generators. The analysis presented is validated by experimental results.

\section{Analysis}

Fig.1 shows $n$ induction machines connected to the same bus across an excitation capacitor $C$ and a load impedance $Z_{L}$. Assume that these $n$ machines are being driven at per unit speeds of $u 1, u 2$, , un, respectively. In parallel operation, all machines will operate at a common terminal voltage and frequency. Figs. 2 shows the per-phase steady-state equivalent circuits of the excitation capacitor and load, and the $i^{\text {th }}$ machine, respectively. In this circuit all the generator parameters are assumed to be constant and are independent of saturation except the magnetizing reactance $X_{m}$. Core losses and effect of harmonics are neglected. 


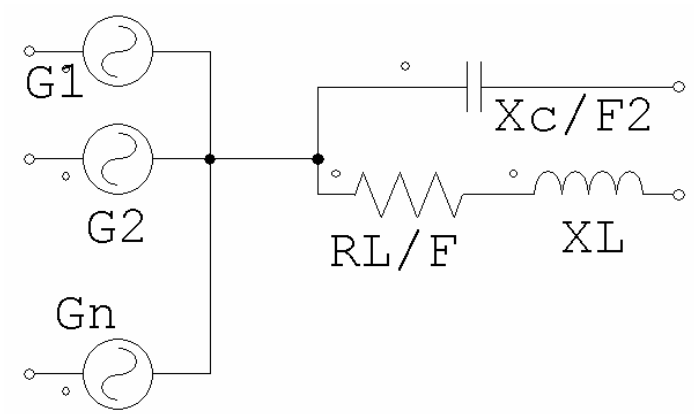

Fig.1 System under study

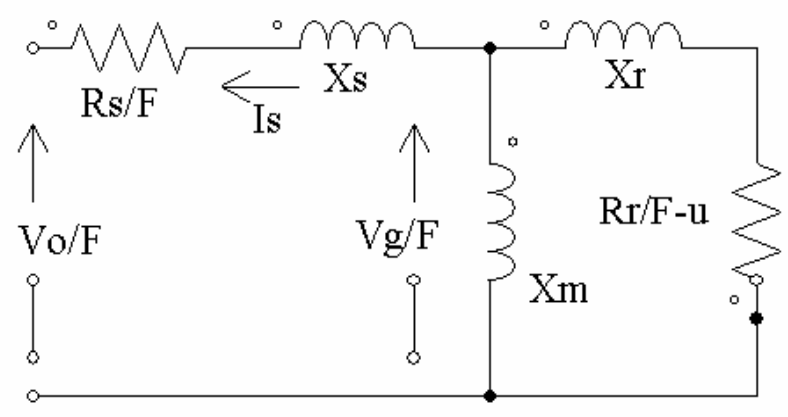

(a)

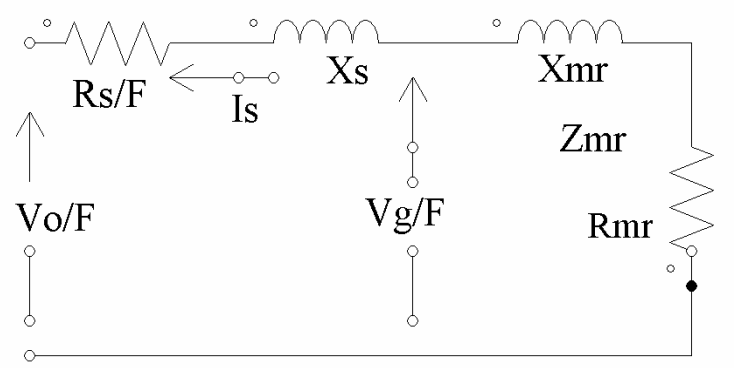

(b)

Fig.2 (a) Equivalent circuit of the $\mathrm{i}^{\text {th }}$ induction generator (b) Simplified circuit of (a)

To represent the parallel operation of these machines, $n$ such equivalent circuits of Fig.2 have to be connected across the load and the excitation capacitor. Steady state balanced equations of the system can be solved for either $F$ and $X_{m i}(\mathrm{i}=1,2 \ldots n), F$ and $X_{c}$, or $F$ and $u_{i}(\mathrm{i}=1,2 \ldots$ $n)$, as shown below.

$$
\left|Z_{i i} I_{s i}\right|-\left|Z_{i n} I_{s n}\right|=0
$$

for $\mathrm{i}=1,2, \ldots .(\mathrm{n}-1) . Z_{i}$ is as given in the Appendix.

From Figs. 2(b), $I_{s i}$ can be expressed as:

$$
I_{s i}=\frac{V_{g i} / F}{Z_{m r i}}
$$

The summation of all the currents injected in the common bus is zero. Accordingly:

$$
\begin{aligned}
& \frac{V_{o} / F}{Z_{i 1}}+\frac{V_{o} / F}{Z_{i 2}}+\ldots . .+\frac{V_{o} / F}{Z_{o}}=0 \\
& \text { or } \\
& Y_{i 1}+Y_{i 2}+\ldots \ldots .+Y_{o}=0
\end{aligned}
$$

where $Y_{i}$ is as given in the Appendix.

\section{A. Solution for $F$ and $X_{m}$}

Eqns. 1-4 are $(n+1)$ nonlinear equations which can be solved for $F$ and $X_{m i}$ for $\mathrm{i}=1,2 \ldots$ n for given values of speeds, $X_{c}$, load and other parameters of each generator.

\section{B. Solution for $F$ and $X_{c}$ for minimum excitation}

When $n$ generators are connected in parallel, any generator will maintain self-excitation if it operates in the saturation region of its magnetization curve. Minimum excitation are sustained when saturation is minimum, i.e. $X_{m i}=X_{o i}$. [3,4]. Only eqn. (4) is to be solved for $F$ and $X_{c}$ to yield the value of $C_{\min }$.

\section{Solution for $F$ and $u$}

Like the case of $F$ and $X_{m}$, eqns. 1-4 are $(n+1)$ nonlinear equations which can be solved for $F$ and $u_{i}$ for $\mathrm{i}=1,2 \ldots . \mathrm{n}$ for given values of $X_{c}, X_{m}$, load and other parameters of each generator.

Once the values of the unknowns are obtained, the complete performance can be easily evaluated by solving the circuit of Fig.1 with the help of the magnetization curve of each generator $[4,7]$.

\section{Proposed Algorithm}

The proposed method to solve for the unknowns in the above formula is a sequential numerical optimizer. This optimizer consists of two parts. First, a simple standard genetic algorithm (GA) is used to bring the values of the unknowns close to the region of the (optimality) solution. Then a classical constrained solver will take over to modify and fine tune the values of these unknowns. This sequential optimizer takes advantage of the capability of GA to locate the region of the optimal solution without being trapped in a local minimum. In addition, it avoids the GA slow convergence. The gradient solver will swiftly converge to the correct solution values since it is properly initialized by the GA close to the solution region.

The first part of the proposed optimizer includes the enumerative heuristic technique. This is represented by a simple GA that has the basic elements of the classical GA. This includes the encoding mechanism, a random method for initialization, the parent selection policy, the mating procedure, and the introduction of mutation as a way to avoid local minima. The second part of the algorithm is a Calculus-based optimizer that employs the gradient-directed searching mechanism. to solve the error surface or differentiable surface of an objective function. For ill-defined or multimodal objective functions, the gradient optimizer is frequently stocked in local minima. However, in the proposed method the GA part will overcome this problem and the calculus-based optimizer will swiftly converge to the correct solution values since it is properly initialized by the GA.

Two schemes have been proposed. First, a scheme of two sequential minimizations is used. A single variable 
minimizer is used to find the value of the frequency $(F)$ then, the same minimizer is used to find the value of the other unknown $\left(u, X_{c}\right.$ or $\left.X_{m}\right)$. A more efficient and reliable scheme which uses several-variable constrained minimization solves the problem directly. This scheme simultaneously finds the values of $X_{c}$ or $X_{m}$ and $F$ that minimize the total impedance of the generator.

\section{Results}

\section{A. Machines under study}

To test the validity of the proposed method of solution, two three phase induction machines were tested. The ratings of these machines are $380 \mathrm{~V}, 1.0 \mathrm{~kW}, 60 \mathrm{~Hz}, 4-$ pole, while the p.u. parameters of them are given in Table no.1. The magnetization curves of the machines are shown in Fig.3. These curves are usually approximated in segments [1-7]. A better way of approximation is by fitting the curves by a polynomial function. Table no.2 shows the coefficients of the machines fitted curves in a polynomial of degree 3.

Table 1: p.u. parameters of G1 and G2

\begin{tabular}{|c|c|c|c|c|}
\hline Generator & $R_{S}$ & $X_{r}=X_{S}$ & $R_{r}$ & $X_{O}$ \\
\hline G1 & 0.1654 & 0.1060 & 0.0932 & 2.84 \\
\hline G2 & 0.0918 & 0.2112 & 0.0635 & 1.95 \\
\hline
\end{tabular}

Table 2: Coefficients of magnetization curve polynomial fitting

\begin{tabular}{|c|c|c|c|c|}
\hline Generator & $k_{o}$ & $k_{1}$ & $k_{2}$ & $k_{3}$ \\
\hline G1 & 1.16143 & 0.308582 & -0.00719 & -0.06382 \\
\hline G2 & 1.90026 & -2.51651 & 2.04632 & -0.5959 \\
\hline
\end{tabular}

\section{B. Experimental Verification}

Fig.4 shows the measured and computed variations of the required minimum excitation capacitance, $C_{\min }$, when G1 and G2 operate in parallel at same speed and under no load. As expected $C_{\min }$ decreases as speed increases. Fig. 5 shows the measured and computed variations of frequency, terminal voltage and stator currents of G1 and G2 operating in parallel at same speed. Fig.6 also shows the same variation of Fig. 5 but when two identical generators of G2 operate in parallel at same speed. The measured results of figures 4, 5 and 6 are quoted from Ref. no. [8]. Good correlation between the computed and experimental results can be noted from these figures.

\section{Conclusions}

In this paper, an optimization-based steady state analysis of parallel operated three phase self-excited induction generators has been introduced. The problem is solved numerically utilizing new optimization algorithms. Instead of step by step analytical derivation of several equations, a global optimizer is used to find the required parameters of the generator. Two methods have been proposed. First, a scheme of two sequential minimizations is used. A single variable minimizer is used to find the value of the frequency $(F)$ then, the same minimizer is used to find the value of the other unknown
( $u, X_{c}$ or $X_{m}$ ). A more efficient and reliable scheme which uses several-variable constrained minimization solves the problem directly. This scheme simultaneously finds the values of $X_{c}$ or $X_{m}$ and $F$ that minimize the total impedance of the generator. The authors of this paper developed a series of integrated program routines to solve the proposed problem using Matlab. In this respect a sequential genetic (GA)/gradient optimizer is used to minimize a cost function of used to solve the total impedance or admittance equations of the circuit of the generator to obtain the frequency and other performance of the machine. Experimental test results confirm the feasibility and accuracy of the proposed scheme.

\section{References}

[1] Bansal, R.C., Bhatti, T.S. and Kothari, D.P., "Bibliography on the Application of Induction Generators in Nonconventional Energy Systems”, IEEE Trans., Vol.EC-18(3), 2003, pp.433439.

[2] Bansal, R.C., "Three-Phase Self-Excited Induction Generators: An Overview”, IEEE Trans., Vol.EC-20(2), 2005, pp.292-299.

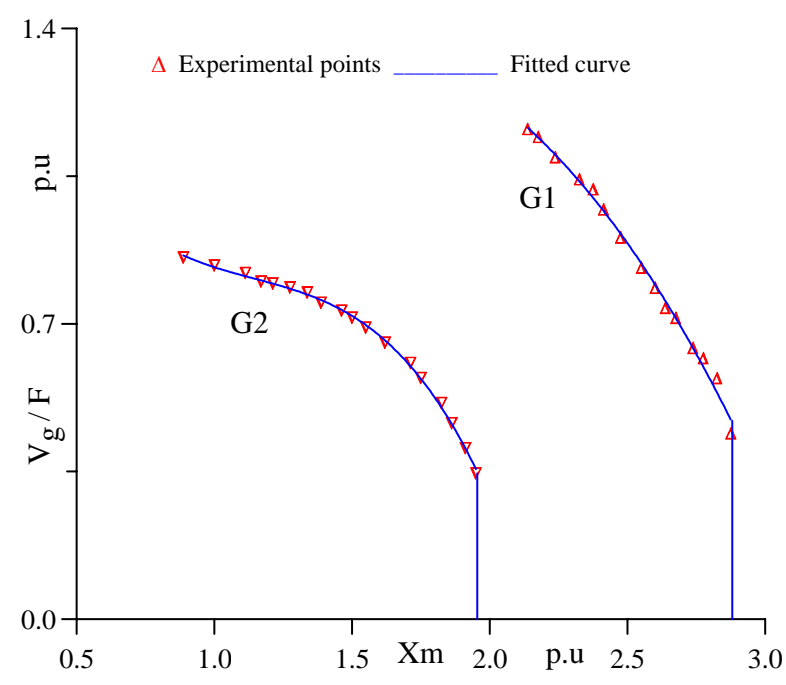

Fig.3 Magnetization curve of G1 and G2

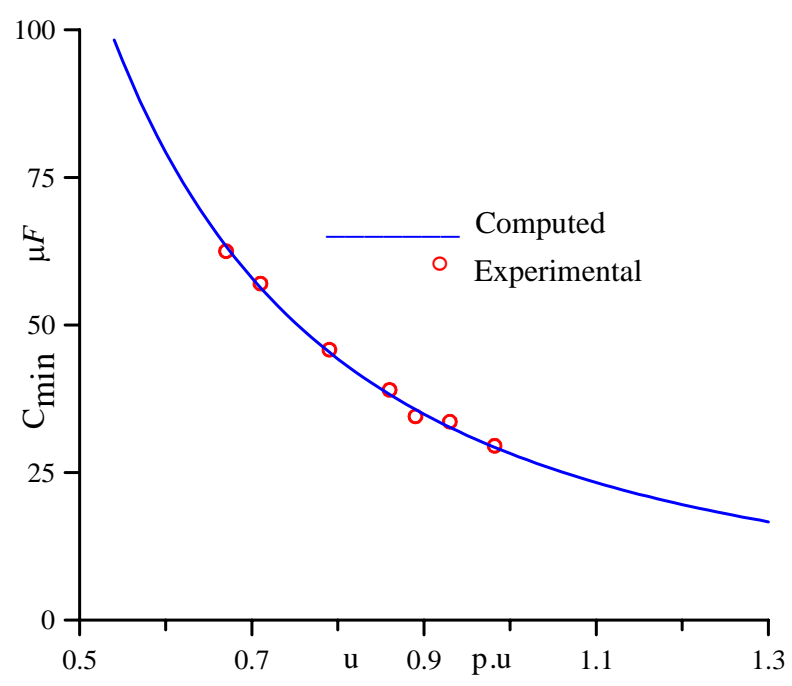

Fig.4 Variation of $C_{\min }$ versus speed for G1// G2 
[3] Aljabri, A.K. and Alolah, A.I. "Capacitance requirement for isolated self-excited induction generator”, Proc. of IEE, pt. B, Vol. 137(3), 1990, pp. 154-159.

[4] Aljabri, A.K. and Alolah, A.I. "Limits on the Performance of Three Phase Self-Excited Induction Generator", IEEE Trans., Vol.EC-5(2), 1990, pp.350-356.

[5] Alolah, A.I., "A Simple Iterative Method for Steady State Analysis of Three Phase Self-Excited Induction Generator", International Conf. on Electrical Machines (ICEM), Istanbul, Turkey, 1998, pp.1550-1553.

[6] Ali, K.F. and Alolah, A.I., "A new approach for the analysis of three phase self-excited induction generators", IEEE IEMD'99 Conference, May 1999, pp.342-344.

[7] Alolah, A.I. and Alkanhal, M.A, "Optimization-based steady state analysis of three phase self-excited induction generator”; IEEE Trans., Vol.EC:-15(1), 2000, pp.61-65.

[8] AI-Bahrani, A.H. and Malik, N.H. "Steady-state analysis of parallel-operated self-excited induction generators”, Proc. of IEE., pt. C, Vol. 140(1), 1993, pp. 49-55.

[9] Wang, C.H. "A Novel Analysis of Parallel Operated SelfExcited Induction Generators”, IEEE Trans., Vol.EC-13(2), 1998, pp.117-123.

[10] Chkraborty, C., Bhadra, S.N. and Chattopadhyay, K. "Analysis of Parallel-Operated Self-Excited Induction Generators”, IEEE Trans., Vol.EC-14(2), 1999, pp.209-216.

\section{Appendix}

$\begin{array}{lll}Z_{s}=R_{S} / F+j X_{s} & Z_{r}=R_{r} /(F-u)+j X_{r} & Z_{m}=j X_{m} \\ Z_{L}=R_{L} / F+j X_{L} & Z_{c}=-j X_{c} / F^{2} & Z_{o}=Z_{m} / / Z_{c} \\ Z_{m r}=Z_{m} / / Z_{r} & Z_{i}=Z_{S}+Z_{m r} & Y_{i}=1 / Z_{i} \\ Y_{o}=1 / Z_{o} & & \end{array}$

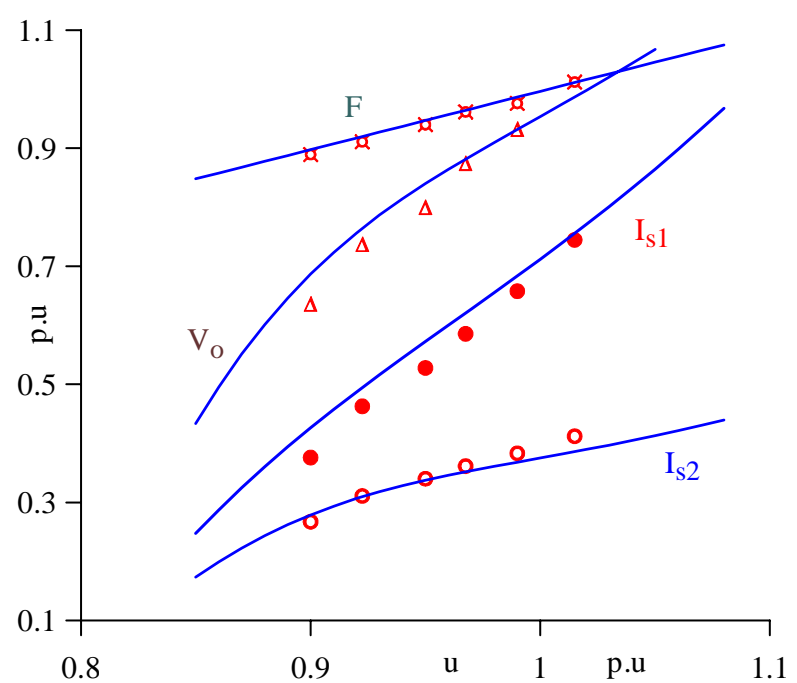

Fig.5 Variation of $F, V_{o}$ and $I_{s}$ versus speed under no load and $C=40 \mu \mathrm{F}$ for $\mathrm{G} 1 / / \mathrm{G} 2$

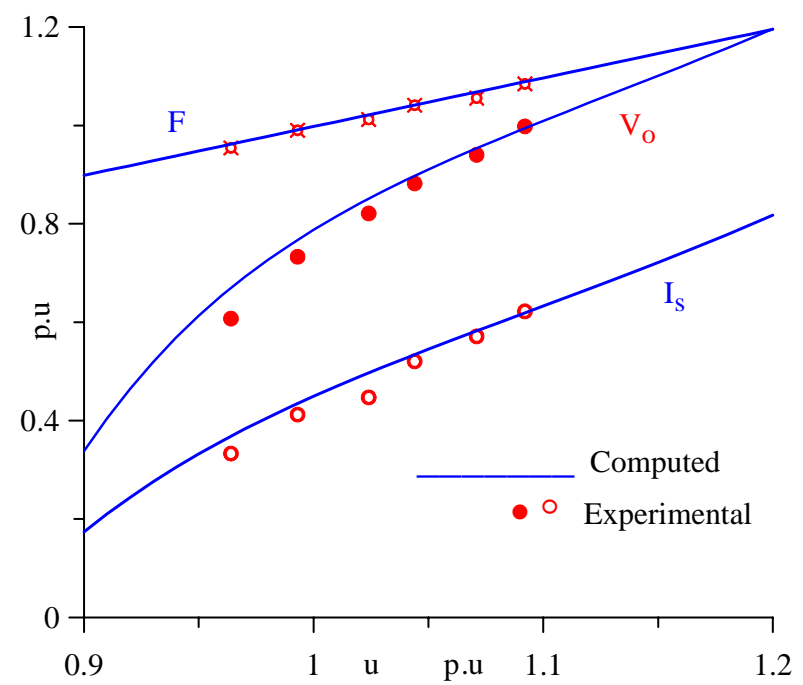

Fig.6Variation of $F, V_{o}$ and $I_{S}$ versus speed under no load and $C=40 \mu \mathrm{F}$ for $\mathrm{G} 2 / / \mathrm{G} 2$ 